\title{
Competencias genéricas de los estudiantes de Auditoría requeridas por las Big Four en Chile*
}

Generic competences of the Audit students required by the Big Four in Chile Competências genéricas dos alunos de Auditoria exigidos pelas Big Four no Chile

\author{
Sebastián Cristóbal Araya-Pizarro \\ Universidad de La Serena, Chile \\ saraya@userena.cl \\ ORCID: http://orcid.org/0000-0002-5857-8441
}

DOI: https://doi.org/10.11144/Javeriana.cc20-49.cgea

Fecha de recepción: 10 Octubre 2018

Fecha de aprobación: 15 Mayo 2019

Fecha de publicación: 30 Junio 2019

\section{Resumen:}

Este artículo analiza las competencias genéricas más valoradas por las cuatro firmas líderes en el sector de la consultoría y auditoría del mundo: las Big Four, con el fin de evidenciar aquellas que deben ser potenciadas en los planes de formación de los estudiantes de contabilidad y auditoría en Chile. Para la recolección de datos se realizaron entrevistas personales a representantes de las empresas, además de la aplicación de cuestionarios a estudiantes que trabajaron en ellas y que fueron seleccionados mediante un muestreo no probabilístico intencional. Los resultados destacan las competencias sistémicas (capacidad de aplicar conocimientos en la práctica, capacidad de motivar, habilidad para trabajar de forma autónoma, capacidad de aprender y actualizarse continuamente), seguidas de competencias interpersonales (trabajo en equipo y habilidades interpersonales), y competencias instrumentales (conocimientos sobre la profesión y capacidad de resolver problemas). Se concluye la necesidad de configurar programas de formación que estimulen el desarrollo de las competencias genéricas deficitarias del estudiante en pro de su mejor inserción y desempeño profesional, bajo un contexto global, dinámico, competitivo y de mayor preocupación social.

Códigos JEL: A22, M41, M42

Palabras clave: Competencias genéricas, contador, auditor, Big Four.

\section{Abstract:}

This article analyzes the most valued generic competences by the four leading firms in the consulting and auditing sector of the world: The Big Four, to highlight those that should be strengthened in the training plans of accounting students and audit in Chile. For the collection of data, personal interviews were conducted with representatives of the companies, in addition to the application of questionnaires to students who worked in them and who were selected under an intentional non-probabilistic sampling. The results highlighting the systemic competences (ability to apply knowledge in practice, ability to motivate, ability to work autonomously, ability to learn and continuously update), followed by interpersonal skills (teamwork and interpersonal skills), and instrumental skills (knowledge about the profession and ability to solve problems). It concludes on the need to configure training programs that stimulate the development of the deficit generic competences of the student in favor of their better insertion and work performance, under a global, dynamic, competitive context, and of greater social concern.

JEL Codes: A22, M41, M42

Keywords: Generic competences, accountant, auditor, Big Four.

\section{Resumo:}

Este artigo analisa as competências genéricas mais valorizadas pelas quatro principais empresas do setor de consultoria e auditoria do mundo: as Big Four, com o objetivo de destacar aquelas que devem ser fortalecidas nos planos de formação de estudantes de contabilidade. e auditoria no Chile. Para a coleta de dados, foram realizadas entrevistas pessoais com representantes das empresas, além da aplicação de questionários aos alunos que nelas trabalhavam e que foram selecionados em uma amostra intencional não probabilística. Os resultados destacam as competências sistêmicas (capacidade de aplicar o conhecimento na prática, capacidade de motivar, capacidade de trabalhar de forma autônoma, capacidade de aprender e atualizar continuamente), seguido de habilidades interpessoais (trabalho em equipe e habilidades interpessoais) e habilidades instrumentais (conhecimento sobre a profissão e capacidade de resolver problemas). Conclui-se sobre a necessidade de configurar programas de treinamento que estimulem o 
desenvolvimento do déficit de competências genéricas do estudante em favor de sua melhor inserção e desempenho no trabalho, sob um contexto global, dinâmico, competitivo e de maior preocupação social.

Códigos JEL: A22, M41, M42

Palavras-chave: Competências genéricas, counter, auditor, Big Four.

\section{INTRODUCCIÓN}

El desarrollo de competencias es uno de los principales desafíos de la educación del siglo XXI (Contreras, 2011; González \& González, 2008), que se ve influenciada al menos por dos grandes procesos revolucionarios: la globalización y la sociedad del conocimiento (Avendaño-Castro, Rueda-Vera, \& PazMontes, 2016).

El fenómeno de la globalización, marcado por la irrupción de las tecnologías de la información y la supresión de las barreras geográficas, ha instaurado el desafío para la Educación Superior de avanzar hacia una formación integral, pertinente a los nuevos requerimientos del mercado laboral y de la sociedad que demandan, entre otros aspectos, la capacidad de desempeñarse en un entorno altamente dinámico y competitivo (Lyudmila, Alexey \& Sevinj, 2019) y bajo una filosofía empresarial diferente, donde la orientación netamente económica ha tenido que ceder lugar al desarrollo de nuevos objetivos en el campo de la responsabilidad social (Pérez, Espinoza, \& Peralta, 2016).

Por su parte, la sociedad del conocimiento experimenta cambios de manera permanente. Las nuevas tecnologías han mejorado significativamente la producción y la difusión del conocimiento, el requisito previo de la innovación. De esta manera, la universidad que ha sido tradicionalmente la principal productora y diseminadora de conocimiento, y la generadora de innovaciones, ha visto afectado su rol, planteándole desafíos que implican reformas notables para satisfacer las demandas imperativas de eficiencia, investigación e innovación (Snellman, 2015). Su adopción significa una valiosa oportunidad de desarrollo humano y compromiso con la equidad (Peralta \& Piña, 2008), y debe concretarse a través de la proyección de un perfil profesional pertinente y acorde a los nuevos requerimientos de las organizaciones del siglo XXI.

De este modo, fomentar el conocimiento, las actitudes y las habilidades de los estudiantes para que puedan trabajar en entornos diferente al propio se ha convertido en una preocupación importante en la sociedad contemporánea (Boateng \& Thompson, 2013), al igual que la incorporación de la Responsabilidad Social en la formación de personas y en las prácticas empresariales (Navarro, Rubio, Lavado, Minnicelli, \& Acuña, 2017), pues se reconoce que para enfrentar exitosamente los retos que impone este nuevo modelo de sociedad compleja, se debe considerar -como relata (Ospina, 2014) - una concepción antropológica más amplia del individuo (cultural, histórica, política y natural), en el que el desarrollo de la persona se distingue en un orden de prelación al profesional (Dextre, 2013).

En el contexto educativo, lo anterior implica, por un lado, dar un nuevo significado a las prácticas de clases para posibilitar la transversalidad de una formación integral bajo una propuesta curricular flexible. Y por otro, evidencia la ineficacia de la mera impartición de conocimientos técnicos, característica de la concepción mecanicista del proceso de enseñanza-aprendizaje tradicional, y conmina invertir tiempo para adquirir nuevas habilidades que posibiliten adaptarse rápidamente a los cambios y obtener buenos resultados (Miyakawa, Kawakubo \& Oguchi, 2019).

Los programas actuales de educación subrayan la formación integral del estudiante, donde un graduado además de aprender los contenidos específicos de su carrera requiere aprender saberes relacionados al trabajo en equipo, la comunicación oral y escrita, la iniciativa y la capacidad de liderazgo, entre otras (Miró \& Jaumei-Capó, 2010). Estas son las denominadas competencias genéricas o transversales, es decir, un conjunto de habilidades no-cognitivas esenciales para aprender y desempeñarse exitosamente en el trabajo (CorominasRovira, 2001). Estas competencias mejoran su valoración en el mundo laboral (Fernández, 2010). No 
obstante, a pesar de que existe un reconocimiento explícito sobre la necesidad por desarrollar ciudadanos con nuevas habilidades y competencias acordes al nuevo paradigma empresarial, aun las instituciones de educación registran importantes deficiencias en su formación (Boude, 2011) sobre todo en aquellas vinculadas a las competencias laborales genéricas (Parlamis \& Monnot, 2018; Villarroel \& Bruna, 2014).

Como indican Montilla \& Herrera (2006), es deber de la auditoría colocar el tono de actualización y optimización en las organizaciones preparándolas para los desafíos de la era moderna, donde las habilidades no cognitivas son consideradas un sustento para el desarrollo integral del ser humano, tanto en lo personal como en lo laboral. En la profesión contable y de auditoría, la adquisición de habilidades genéricas es de vital importancia dado que los profesionales tienen que competir en un mercado globalizado y competitivo dominado por los avances en la tecnología de la información y las comunicaciones (Bunney, Sharplin \& Howitt, 2015; Carter \& Spence, 2014; Crawford, Helliar \& Monk, 2011).

Por lo tanto, es posible apreciar que existen diversos riesgos y retos que la globalización le presenta a la formación del Contador chileno y latinoamericano, y que solo pueden enfrentarse, conviniendo con lo destacado por Dextre (2013), mediante una formación de calidad capaz de satisfacer los requerimientos internacionales de conocimientos, habilidades y virtudes. Por ejemplo, Bunney et al. (2015) realizan un interesante análisis sobre las habilidades genéricas en contadores graduados en el que señalan que el papel de las universidades, en la actualidad, se extiende más allá del imperativo económico clásico para abarcar un mayor papel social y cultural. Argumentan que la nueva economía del conocimiento necesita graduados con una mentalidad flexible y habilidades transferibles, capaces de innovar y adaptarse a un entorno de trabajo dinámico.

Las Big Four o Big 4 es el término inglés utilizado para referirse a las cuatro firmas de contabilidad y auditoría más grandes del mundo y que se encuentran también presentes en Chile: Deloitte, PriceWaterhouseCoopers, Ernst \& Young y KPMG. Estas empresas ofrecen una amplia gama de servicios de contabilidad y auditoría, incluyendo auditoría externa, servicios de asesoramiento fiscal, gestión y consultoría de negocios, la evaluación de riesgos y control, y en los últimos años han aumentado su participación en el mercado de aseguramiento de la sustentabilidad (Fernandez-Feijoo, Romero, \& Ruiz, 2018).

Con este contexto, la presente investigación analiza las competencias genéricas que debe poseer un auditor para obtener un buen desempeño laboral en las empresas consultoras más exitosas del mundo, contribuyendo así a descubrir los aspectos claves de la formación integral de profesionales capaces de cumplir con las expectativas del mercado, y como consecuencia, ayudar además a mejorar el desempeño y la empleabilidad de los estudiantes, que en el caso de la unidad de estudio (Universidad de La Serena) registra tasas por debajo del promedio de las 10 principales universidades de Chile (81,9\% vs 95,6\%).

A continuación, se presenta un breve resumen teórico de las competencias genéricas en la formación universitaria, luego se exponen los aspectos metodológicos del diseño del estudio, para posteriormente presentar los principales hallazgos, y finalizar con las conclusiones del estudio.

\section{LAS COMPETENCIAS GENÉRICAS EN LA FORMACIÓN UNIVERSITARIA}

La evolución desde el modelo clásico fordista-taylorista de producción al modelo económico de la tecno-globalización, la economía informacional y la globalización de los mercados ha llevado a que las organizaciones deban adaptarse a las nuevas dinámicas sociales y económicas. Esto les exige contar con personas que sepan desempeñarse en organizaciones flexibles, relacionales, globales, planas y diversas (Abarca, 2016), mediante la adaptación de un nuevo paradigma de gestión empresarial enfocado en el aumento de competencias cognitivas, habilidades instrumentales y valores de sus colaboradores (Dextre, 2013). 
Una competencia es un saber hacer complejo, que se manifiesta en la actuación efectiva sobre una situación problemática, para cuya solución se movilizan integradamente diferentes habilidades, conocimientos y actitudes (Camargo-Escobar \& Pardo-Adames, 2008; Rodríguez, 2007). Se dice que las competencias son características o habilidades únicas de un individuo relacionadas con un desempeño laboral superior (Shah \& Prakash, 2018). Para Tobón (2005) este término representa la orientación del desempeño humano hacia la idoneidad en la realización de actividades y resolución de problemas. Y que, al igual que la inteligencia, es susceptible de ser adquirida a través de la enseñanza (Bautista, 2016) y construida a partir de las motivaciones internas del individuo (Beneitone et al., 2007).

De acuerdo con Roncancio, Mira y Muñoz (2017) una de las clasificaciones para las competencias que goza de mayor grado de consenso entre autores y la IFAC (International Federation of Accountans) es aquella que las divide en competencias básicas o instrumentales, competencias genéricas o transversales y competencias específicas o técnicas (tabla 1 ).

TABLA 1

Tipos de competencia según grado

\begin{tabular}{|l|l|}
\hline Competencia & Descripción \\
\hline $\begin{array}{l}\text { Básicas o } \\
\text { Instrumentales }\end{array}$ & $\begin{array}{l}\text { Son las asociadas a conocimientos fundamentales que, } \\
\text { normalmente se adquieren en la formación general y permiten el } \\
\text { ingreso al trabajo. }\end{array}$ \\
\hline $\begin{array}{l}\text { Genéricas o } \\
\text { Transversales }\end{array}$ & $\begin{array}{l}\text { Son las relacionadas con los comportamientos y actitudes de las } \\
\text { labores propias de diferentes ámbitos de producción. }\end{array}$ \\
\hline Específicas o Técnicas & $\begin{array}{l}\text { Son las relacionadas con aspectos técnicos directamente } \\
\text { vinculados con la ocupación y que no son tan fácilmente } \\
\text { transferibles a otros contextos laborales. }\end{array}$ \\
\hline
\end{tabular}

Fuente: Roncancio, Mira \& Muñoz (2017).

De esta manera, las competencias básicas son las competencias fundamentales para vivir en sociedad y desenvolverse en cualquier ámbito laboral. Las competencias específicas son aquellas competencias propias de una determinada ocupación o profesión y que se caracterizan por tener un alto grado de especialización y, finalmente, las competencias genéricas son aquellas competencias comunes a varias ocupaciones o profesiones y se entienden como fundamentales para que un individuo pueda afrontar los continuos cambios del quehacer profesional (Corominas-Rovira, 2001).

Rychen y Salganik (2003) citados por Villarroel \& Bruna (2014) señalan que existen cuatro elementos analíticos que definen una competencia genérica: a) son transversales en diferentes campos sociales, afectando a varios sectores de la existencia humana, b) aluden a un orden superior de complejidad mental, favoreciendo el desarrollo de niveles de pensamiento intelectual de orden superior como son el pensamiento crítico y analítico, reflexión y autonomía mental; c) son multifuncionales, por lo que requieren en un campo extenso y diverso de demandas cotidianas, profesionales y de la vida social; d) son multidimensionales, pues consideran dimensiones perceptivas, normativas, cooperativas y conceptuales, entre otras.

En tal dirección, el proyecto Alfa Tuning se refiere a las competencias genéricas como competencias transferibles, necesarias para el empleo y la vida como ciudadano responsable; siendo relevantes para todos los educandos trascendiendo así la disciplina que se curse (Villarroel \& Bruna, 2014). Estas abarcan una combinación dinámica de conocimiento, comprensión, capacidades y habilidades (Cabeza, Castrillón, \& Lombana, 2015), que ponen de manifiesto la capacidad de una persona para desempeñarse en tareas profesionales (Kallioinen, 2010).

Son cuantiosas las clasificaciones de competencias laborales genéricas que han identificado diferentes autores en su búsqueda de aquellas que responden, en mayor medida, a los requerimientos emanados del desempeño laboral. Por ejemplo, el modelo propuesto por Spencer \& Spencer (1993) citado por Gil 
(2007) recoge seis grupos de competencias genéricas: competencias de desempeño y operativas, competencias de ayuda y servicio, competencia de influencia, competencias directivas, competencias cognitivas, y competencias de eficacia personal (tabla 2).

TABLA 2

Clasificación de las competencias laborales genéricas de Spencer \& Spencer (1993)

\begin{tabular}{|c|c|}
\hline Grupos & Competencias genéricas \\
\hline $\begin{array}{l}\text { Competencias de desempeñoy } \\
\text { operativas }\end{array}$ & $\begin{array}{l}\text { - Orientación al resultado } \\
\text { - Atención al orden, calidad y perfección } \\
\text { - Espintu de iniciativa } \\
\text { - Búsqueda de la información }\end{array}$ \\
\hline Competencias de ayuda y servicio & $\begin{array}{l}\text { - Sensibilidad interpersonal } \\
\text { - Orientación al cliente }\end{array}$ \\
\hline Competencias de influencia & $\begin{array}{l}\text { - Persuasión e influencia } \\
\text { - Conciencia organizativa } \\
\text { - Construcción de relaciones }\end{array}$ \\
\hline Competencias directivas & $\begin{array}{l}\text { - Desarrollo de los otros } \\
\text { - Actitudes de mando: asertividad y uso del poder formal } \\
\text { - Trabajo en gupo y cooperación } \\
\text { - Liderazgo de grupos }\end{array}$ \\
\hline Competencias cognitivas & $\begin{array}{l}\text { - Pensamiento analítico } \\
\text { - Pensamiento conceptual } \\
\text { - Capacidades técnicas, profesionales y directivas }\end{array}$ \\
\hline Competencias de eficacia personal & $\begin{array}{l}\text { - Autocontrol } \\
\text { - Confianza en sí mismo } \\
\text { - Hexibilidad } \\
\text { - Hábitos de organización }\end{array}$ \\
\hline
\end{tabular}

Fuente: Gil (2007).

El proyecto Alfa Tuning América Latina identifica 27 competencias genéricas comunes a cualquier titulación y las agrupa en tres clasificaciones: competencias instrumentales que son consideradas como medios para obtener un determinado fin (capacidades cognitivas, metodológicas, tecnológicas y lingüísticas: capacidad de análisis y síntesis, capacidad de organización y planificación, conocimientos generales básicos, conocimientos básicos de la profesión, comunicación oral y escrita, habilidades de manejo de un computador, habilidades de gestión de la información, resolución de problemas, toma de decisiones); competencias interpersonales, que se refieren a las diferentes capacidades que hacen que las personas logren una buena interacción con los demás (capacidades individuales tales como habilidades sociales: capacidad crítica y autocrítica, trabajo en equipo, habilidades interpersonales, trabajo en equipo interdisciplinario, capacidad de comunicarse con expertos de otras áreas, reconocimiento de la diversidad y multiculturalidad, habilidad para trabajar en un contexto internacional, compromiso ético), y competencias sistémicas, relacionadas con la comprensión de la totalidad de un conjunto o sistema (capacidades y habilidades relacionadas con sistemas globales: capacidad de aplicar los conocimientos en la práctica, habilidades de investigación, aprendizaje, adaptación a nuevas situaciones, creatividad, liderazgo, conocimiento de culturas y costumbres de otros países, habilidad de trabajar de manera autónoma, diseño y gestión de proyectos, iniciativa y espíritu emprendedor, motivación por la calidad, motivación por la consecución de objetivos) (Amor \& Serrano, 2019; Beneitone et al., 2007; Esquetini et al., 2013). Todas las competencias se presentan en la tabla 3. 
TABLA 3

Competencias genéricas del modelo Tuning América Latina

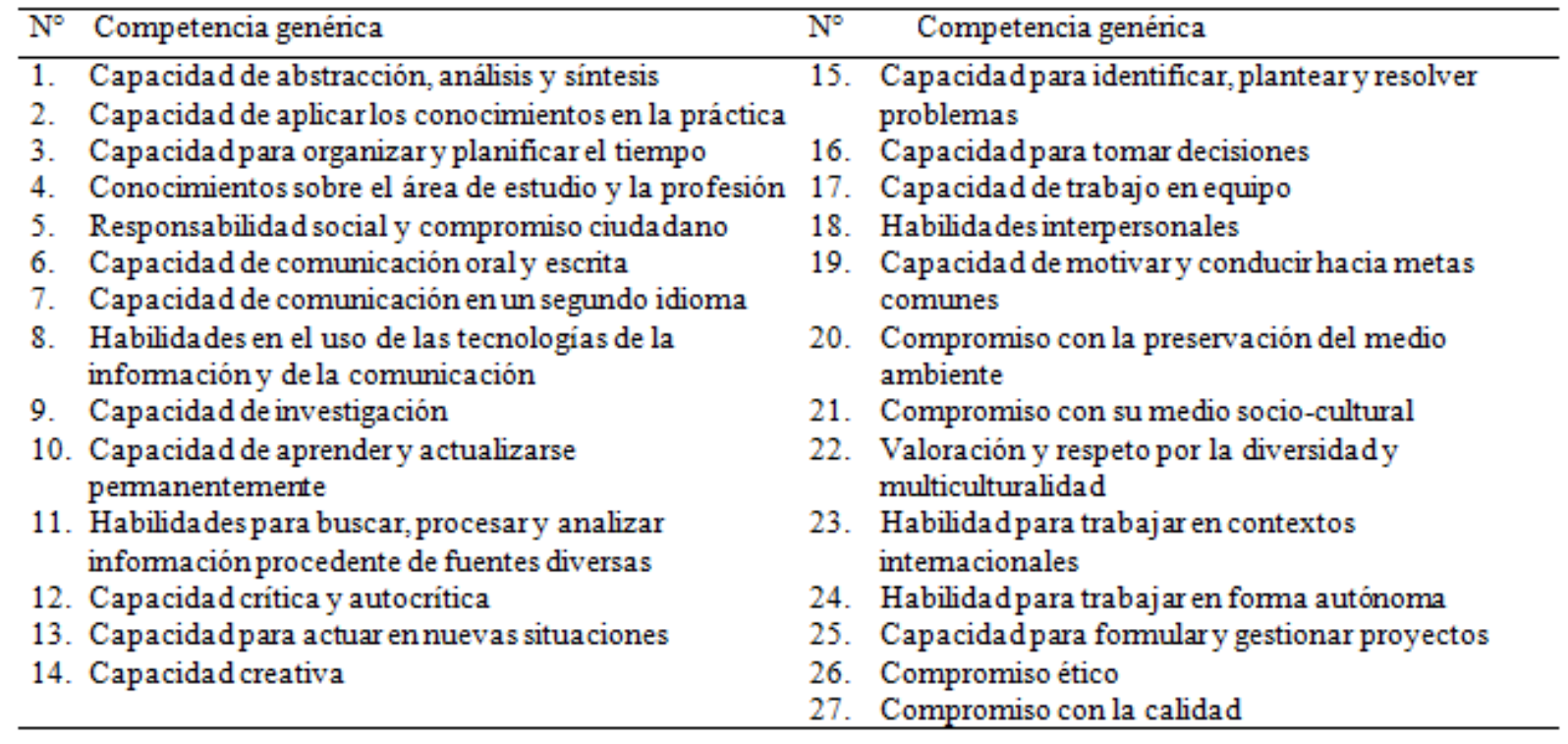

Fuente: elaboración propia en base a Beneitone et al. (2007).

También la OCDE (2001) construyó un modelo de competencias, denominado DeSeCo (Definition and Selection of Competences) proyecto encargado de definir y seleccionar las competencias consideradas esenciales para la vida de las personas y el buen funcionamiento de la sociedad (key competences). En él se clasificaron las competencias en tres grandes categorías (interactuar con grupos sociales heterogéneos, actuar con autonomía y usar las herramientas de forma interactiva), desde donde luego se fijaron las que consideran las ocho competencias claves o básicas para cualquier individuo: a) comunicación en lengua materna, b) comunicación en lengua extranjera, c) conocimientos matemáticos básicos, d) competencias básicas en ciencia y tecnología, e) competencia digital,f) aprender a aprender, g) competencias interpersonales y cívicas, h) emprendimiento y expresión cultural (de Pablos, 2010; Ureña \& Ureña, 2016; Villarroel \& Bruna, 2014). Sin embargo, este proyecto tiene un origen y propósito diferentes al de la definición de perfiles profesionales, como lo realizado por el proyecto Tuning, sino que está más comprometido con garantizar la equidad y la ciudadanía (López, Benedito \& León, 2016).

Al considerar la relación de las competencias con los puestos específicos de trabajo (y naturaleza de la organización), es que se torna necesario que dichas competencias se diversifiquen y adapten a las funciones y tareas específicas definidas para los mismos. Villarroel \& Bruna (2014) sintetizaron diferentes experiencias que revelan lo complejo que es llegar a un acuerdo respecto a las competencias genéricas fundamentales. Entre sus resultados destacaron, a modo de ilustración, las competencias preferidas por profesores y estudiantes de México (capacidad de aplicar los conocimientos a la práctica, la capacidad para identificar, planear y resolver problemas, y la capacidad de aprender y actualizarse permanentemente), Japón (la resolución de problemas, comunicación oral, precisión y atención al detalle), Inglaterra (trabajo bajo presión, comunicación oral, precisión y atención al detalle) y de otros países europeos (resolución de problemas, trabajo autónomo, comunicación oral). En esta dirección, la IFAC plantea que el objetivo principal de la educación en Contaduría es desarrollar profesionales competentes con la habilidad de desempeñar su rol de trabajo cumpliendo los estándares que el ambiente laboral demanda, demostrando conocimiento de la profesión, habilidades, valores, ética y actitudes suficientes para hacer frente a un ambiente complejo (Durán, Mejía \& Reyes, 2012).

En Chile, la mayoría de los modelos educativos se basan en modelos internacionales, como el Programa Tuning Europeo o su adaptación a Latinoamérica, que si bien tiene la ventaja de adecuar competencias con 
evidencia de validez empírica, igualmente deriva en el problema de la falta de contextualización a la realidad local. Así mismo, deja importantes cuestionamientos, como por ejemplo: ¿de qué manera deben enseñarse?, ¿cómo deben evaluarse?, ¿de cuáles competencias se puede hacer cargo un plantel educativo?, ¿existen competencias con mayor transversalidad que otras?, etc. También, cabe preguntarse, si hay una mezcla de competencias genéricas que están más relacionadas con algunas carreras que otras, y que conllevarían a una selección más precisa al perfil profesional respectivo.

En conclusión, las categorizaciones presentadas se transforman en proposiciones bastante generales en muchos aspectos. Por tanto, cualquier plantel interesado en incluir en su proyecto educativo competencias genéricas, tiene el reto de estudiar qué competencias genéricas potenciar dentro del marco propio de su campo de acción (Villarroel \& Bruna, 2014).

\section{METODOLOGÍA}

La investigación, desarrollada bajo un paradigma interpretativo y de enfoque mixto, utilizó un diseño de triangulación concurrente, donde se recopilaron datos cualitativos y cuantitativos simultáneamente con el propósito de identificar las competencias genéricas de los contadores auditores requeridas por las 4 grandes auditoras del mundo (Deloitte, PwC, EY y KPMG), presentes en Chile (tabla 4).

TABLA 4

Las Big Four

\begin{tabular}{|l|l|}
\hline Auditora & Descripción \\
Deloitte & $\begin{array}{l}\text { Fue fundada en } 1845 \text { y tiene su sede central en Nueva York, Estados Unidos. } \\
\text { Cuenta con más de } 240.000 \text { profesionales en } 150 \text { países de todo el mundo. } \\
\text { Presta servicios en torno a cinco áreas funcionales: consultoría, impuestos, } \\
\text { asesoria juridica, asesoria financiera y auditoría. }\end{array}$ \\
\hline PwC & $\begin{array}{l}\text { PriceWaterhouseCoopers nació en } 1849 \text { y tiene su sede central en Londres, Reino } \\
\text { Unido. Cuenta con más de } 200.000 \text { trabajadores y opera en más de 158 paises. } \\
\text { Entre sus servicios destacan la auditoria, el asesoramiento legal y fiscal, la } \\
\text { Consultoria de negocio, las transacciones, y el corporate finance. }\end{array}$ \\
\hline KPMG & $\begin{array}{l}\text { Ernst \& Young fue fundada en } 1989 \text { y tiene su sede central en Londres, Reino } \\
\text { Unido. Cuenta con más de } 190.000 \text { empleados que incluyen auditoría, } \\
\text { impuestos, finanzas, contabilidad, servicios de cálculos y estudios actuariales y } \\
\text { asesoramiento en la gestión de la empresa. }\end{array}$ \\
\hline & $\begin{array}{l}\text { Fue fundada en 1917 y tiene su sede central en Amstelveen, Países Bajos. Es una } \\
\text { red global de firmas de servicios profesionales que cuenta con más de 120.000 } \\
\text { empleados y opera en más de } 156 \text { países en el campo de la auditoria, fiscalidad } \\
\text { yasesoramiento financiero y de negocio. }\end{array}$ \\
\hline
\end{tabular}

Fuente: elaboración propia (2018).

Los datos cualitativos fueron recolectados por medio de entrevistas a los representantes de las distintas auditoras que conforman The Big Four, a quienes se les aplicó una entrevista personal, semi-estructurada, que constó de una guía de 26 preguntas abiertas (de opinión, de antecedentes y de simulación), cuyas respuestas fueron registradas por escrito. El propósito de la entrevista fue identificar lo que requiere, en términos de competencias genéricas, una auditora de un egresado de la carrera de auditoría.

Además, para la recolección de datos cuantitativos se aplicaron, bajo un muestreo no probabilístico intencional, 17 encuestas on-line a estudiantes egresados de la carrera de auditoría de la Universidad de La Serena (ULS), única universidad pública de la Región de Coquimbo, Chile, que realizaron práctica profesional en las Big Four durante el periodo 2011-2016. El cuestionario elaborado constó de diez preguntas cerradas y una pregunta abierta, y tuvo por finalidad conocer las principales competencias exigidas en el desarrollo de su práctica profesional, a objeto de contrastar y verificar sus respuestas con lo declarado por los 
empleadores de las auditoras bajo estudio. La pauta de entrevista y el cuestionario fueron validados por el juicio de tres expertos (un metodólogo, el director de la carrera de Auditoría de la ULS y un académico con especialidad en liderazgo, dirección estratégica y comunicación en las organizaciones).

El análisis cualitativo de los datos incluyó el análisis de contenido (categórico descriptivo) por medio de la codificación y categorización de las entrevistas, las cuales fueron, posteriormente, analizados y presentados en cuadros y gráficas radiales que sintetizaron los principales constructos bajo estudio. Mientras que para el análisis cuantitativo de datos se construyeron tablas de frecuencias y gráficas de barras, utilizando el software estadístico SPSS, versión 24 para MS Windows. La figura 1 sintetiza la metodología usada en el estudio: las dos etapas concurrentes (una fase cualitativa y otra cuantitativa) y los tres procesos claves desarrollados (recolección de datos, análisis de las entrevistas y de los cuestionarios e integración).

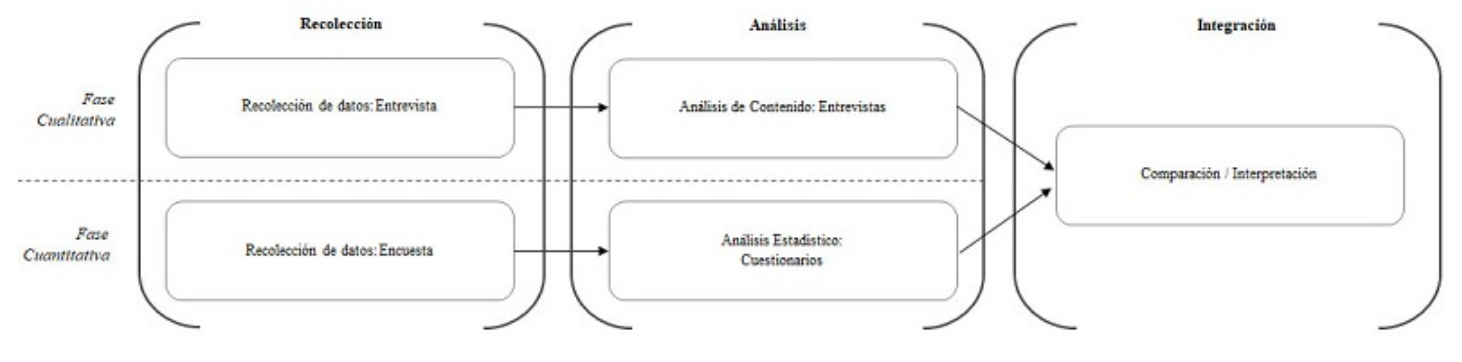

FIGURA 1

Fases de investigación del estudio

Fuente: elaboración propia.

La etapa inicial comienza con la selección de las fuentes de información y la subsiguiente recolección de los datos de las entrevistas y encuesta. En la etapa de análisis se realiza el examen de los datos cualitativos y cuantitativos que permite identificar la información relevante para el estudio. En la dimensión cualitativa se analizan cada uno de los relatos recabados para seleccionar y conceptualizar los significados que el texto contiene dividiéndolos en fragmentos que son luego clasificados en categorías. Posteriormente estos son medidos a través del cómputo de frecuencias y representados gráficamente. En la fase cuantitativa se organizan las respuestas de los encuestados en tablas de frecuencias absolutas y relativas, y culmina con su representación gráfica. Y finalmente, en la última etapa se lleva a cabo el proceso de integración de los análisis, en la cual se contrasta la información de todos los participantes y logra formularse una respuesta global a la problemática planteada.

\section{RESULTADOS Y DISCUSIÓN}

A continuación se presentan los resultados del estudio divididos en tres secciones. Primero se sintetizan las competencias genéricas claves declaradas por las empresas auditoras de sus colaboradores. Segundo, se expone la percepción de los egresados de la carrera de Auditoría de la ULS (que se desempeñaron en las Big Four) sobre los conocimientos y habilidades adquiridas en su formación profesional y laboral en la universidad. Y tercero, se triangulan los resultados previos mediante el contraste de sus testimonios.

\section{Competencias genéricas requeridas por las Big Four}

Las competencias genéricas señaladas por cada una de las firmas entrevistadas, se exponen poniendo énfasis en la identificación de aquellas habilidades y capacidades consideradas necesarias para ser contratados como asistentes B (primer cargo que se opta al ingresar a una auditora), y que completan los conocimientos sobre el área de estudio y la profesión y la capacidad para aplicarlos en la práctica, que fueron, inconcusamente, mencionados por todas las auditoras (tabla 5). 
TABLA 5

Resumen de los requerimientos más destacados que buscan las auditoras

\begin{tabular}{|c|c|c|c|}
\hline Deloitte & PWC & KPMG & EY \\
\hline $\begin{array}{l}\text { - Contar conlos } \\
\text { conocimientosy } \\
\text { capacidad de aplicarlos } \\
\text { - Saber trabajar bajo } \\
\text { presión } \\
\text { - Autonomía } \\
\text { - Trabajo en equipo } \\
\text { - Capacidad de } \\
\text { comunicaruna imagen } \\
\text { profesional } \\
\text { - Habilidad para } \\
\text { enfrentary solucionar } \\
\text { problemas } \\
\text { - Motivación } \\
\text { - Compromiso por } \\
\text { aprender } \\
\text { - Flexibilidad } \\
\text { - Capacidad de } \\
\text { desenvolverse ante } \\
\text { contextos complejos }\end{array}$ & $\begin{array}{l}\text { - Contar con los } \\
\text { conocimientosy } \\
\text { capacidad de aplicarlos } \\
\text { - Comprometidos con su } \\
\text { aprendizaje continuo. } \\
\text { - Habilidad para } \\
\text { solucionar problemas } \\
\text { - Motivación } \\
\text { - Proactividad } \\
\text { - Trabajo en equipo } \\
\text { - Capaz de ayudar a } \\
\text { otros. } \\
\text { - Capacidad de } \\
\text { desenvolverse ante } \\
\text { contextos complejos }\end{array}$ & $\begin{array}{l}\text { - Contar con los } \\
\text { conocimientosy } \\
\text { capacidad de aplicarlos } \\
\text { - Motivación } \\
\text { - Ganas de aprender } \\
\text { constantemente } \\
\text { - Empáticos con el } \\
\text { equipo de trabajo } \\
\text { - Compromiso con su } \\
\text { trabajo } \\
\text { - Comprometidos con } \\
\text { seguir aprendiendo } \\
\text { - Proactividad }\end{array}$ & $\begin{array}{l}\text { - Contar conlos } \\
\text { conocimientosy } \\
\text { capacidad de aplicarlos } \\
\text { - Integridad } \\
\text { - Respeto } \\
\text { - Trabajo en equipo } \\
\text { - Gente con energía } \\
\text { - Entusiasmo } \\
\text { - Liderazgo }\end{array}$ \\
\hline
\end{tabular}

Fuente: elaboración propia.

Primeramente, Deloitte declaró que para poder trabajar como colaborador en la firma es necesario poseer habilidades para desempeñarse bajo presión, habilidad para trabajar en forma autónoma y con motivación y capacidad de trabajo en equipo, además de la flexibilidad que exige desempeñar un cargo como asistente B (capacidad para actuar en nuevas situaciones). La empresa presta especial atención a la capacidad de la persona para identificar y solucionar los problemas que se presentan en la interacción con los clientes. Para ello, indican- es menester que el auditor tenga la capacidad de desenvolverse en escenarios complejos y demuestre desplante para comunicar una imagen profesional.

PriceWaterhouseCoopers señaló valorar trabajadores que se encuentren altamente motivados y comprometidos con su aprendizaje (capacidad de aprender y actualizarse permanentemente). La firma necesita personas que se muestren proactivos en sus funciones, lo que implica tener la capacidad de plantear y resolver problemas, pedir ayuda cuando sea necesario y ser capaz de ayudar a otros (habilidades interpersonales). Un elemento clave es que la persona desee hacer carrera en la firma y estar dispuesto a trabajar para esto, aprendiendo más sobre el rubro de auditoría (capacidad de aprender y actualizarse permanentemente). PwC destaca que las habilidades que se necesitan para ascender a senior (uno de los cargos de trabajo con mayores responsabilidades dentro de la Auditoría) son buenas habilidades para relacionarse y liderazgo, compromiso y trabajo en equipo (capacidad de motivar y conducir hacia metas comunes).

KPMG reveló que los auditores deben tener motivación y ganas de aprender, personas empáticas con el equipo de trabajo, con capacidad para identificar y solucionar problemas y pedir ayuda cuando sea necesario. Además, destacan que para que un solicitante pase a formar parte del equipo KPMG debe ser un profesional comprometido con las tareas asignadas, con motivación para seguir aprendiendo, manifestando proactividad y ganas de superación (capacidad para tomar decisiones, capacidad de aprender y actualizarse permanentemente). Ernst \& Young, por su parte, destacó la integridad de los profesionales auditores (compromiso ético, compromiso con su medio sociocultural), que demuestren respeto y capacidad de trabajo en equipo, profesionales con energía, entusiasmo y capaces de liderar equipos de trabajo (capacidad de aprender y actualizarse permanentemente). 
De este modo, bajo la perspectiva del proyecto Tuning para América Latina, las auditoras acentuaron principalmente once competencias genéricas fundamentales: conocimientos sobre el área de estudio y la profesión, capacidad para aplicar los conocimientos en la práctica, capacidad de trabajo en equipo, capacidad de motivar y conducir hacia metas comunes, habilidades interpersonales, capacidad para identificar, plantear y resolver problemas, habilidad para trabajar en forma autónoma, capacidad de aprender y actualizarse permanentemente, capacidad de comunicación oral y escrita, capacidad para actuar en nuevas situaciones y compromiso con la ética (figura 2).

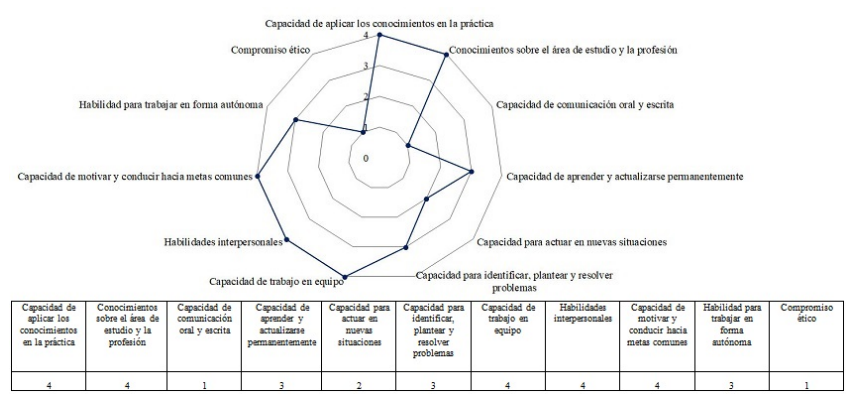

FIGURA 2

Competencias genéricas destacadas por las Big Four, bajo el modelo Tuning Latino América Fuente: elaboración propia.

Cabe destacar que la revisión de los resultados bajo el modelo de las competencias laborales genéricas de Spencer \& Spencer (1993), denota una convergencia plena con lo expuesto anteriormente, donde las Big Four destacaron los grupos de competencias conocidas como: competencias cognitivas (capacidades técnicas, profesionales y directivas y pensamiento analítico), competencias directivas (trabajo en grupo y cooperación y liderazgo de grupos), competencia de eficacia personal (autocontrol, confianza en sí mismo, flexibilidad y hábitos de organización) y competencias de desempeño y operativas (espíritu de iniciativa y atención al orden, calidad y perfección). (figura 3 ).

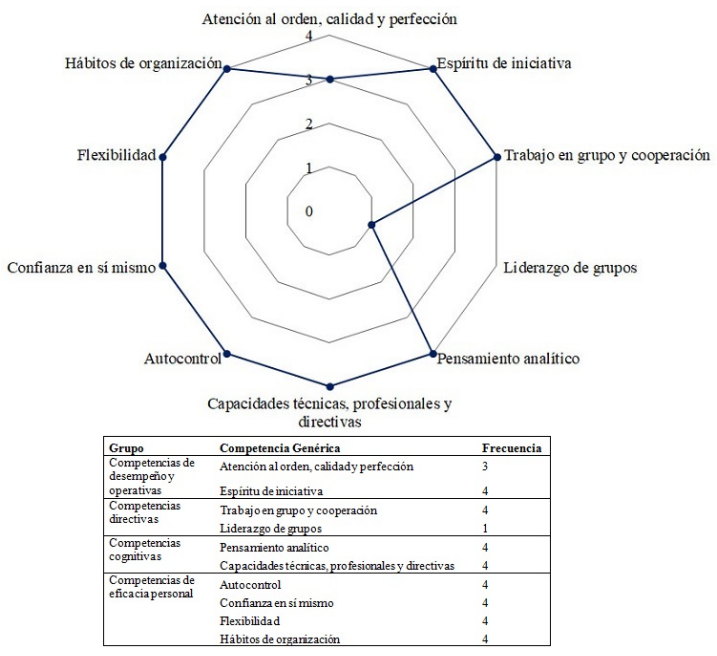

FIGURA 3

Competencias genéricas destacadas por las Big Four, bajo el modelo de Spencer \& Spencer Fuente: elaboración propia.

Finalmente, también se les consultó a las auditoras respecto del énfasis otorgado a las competencias técnicas, a lo que respondieron que estas responden más bien a un estándar para la selección del cargo (contar con los conocimientos suficientes para cumplir las funciones encomendadas), pero que no es lo más relevante para ingresar y permanecer en sus organizaciones. Esto debido a que las grandes auditoras tienen programas de capacitación continua para el desarrollo profesional de sus colaboradores y poseen convenios con diversas instituciones educativas destinadas para su formación en temas de la especialidad. Por ello, recalcan que lo sustancial es contar con personas con capacidades que van más allá de lo meramente intelectual, coincidiendo 
con lo expuesto por muchos autores, que acentúan la relevancia de las competencias genéricas (Fernández, 2010; González \& González, 2008; Rubau, Tovar, \& Fernández, 2013; Tejeda, 2017; Ureña \& Ureña, 2016).

\section{Percepción de los egresados de la carrera de Auditoría}

Los resultados obtenidos de la encuesta aplicada a los egresados de la carrera de Auditoría de la ULS permitieron constatar el acuerdo unánime respecto de la relevancia que tienen las competencias genéricas para el adecuado desempeño laboral de los contadores auditores al interior de las Big Four. De ellas destacaron las competencias vinculadas a conocimientos sobre la profesión y la capacidad para aplicar los conocimientos en la práctica, cuya valoración fue muy superior a la de cualquier otra competencia genérica, como el trabajo en equipo, la autonomía, la capacidad de resolver problemas y la capacidad de adaptarse a situación complejas, las que identificaron como complementarias (aunque esenciales) para desarrollar un trabajo en armonía y mantener un buen clima laboral.

$\mathrm{Al}$ ser consultados en cuanto a si la universidad potencia dichas competencias genéricas, un 23,5\% indicó que la carrera de Auditoría no contribuye mayormente a su desarrollo, lo que dejaría de manifiesto la supremacía de un modelo más académico de formación, centrado en el "saber" que en el "saber hacer" y "saber ser” (Tobón, 2005). Y por tanto instauraría el desafío, como señalan algunos autores, de configurar prácticas pedagógicas más integradoras y holísticas que subrayen el valor de desarrollar competencias instrumentales, interpersonales y sistémicas (Rullan, Fernández, Estapé, \& Márquez, 2010). Estas consideraciones encuentran coincidencia con muchas de las críticas vertidas sobre la formación universitaria actual, donde se demanda mayor atención al contexto social, más compromiso con la formación práctica y la necesidad de abrirse a nuevas metodologías basadas en la resolución de problemas, aprendizaje cooperativo o al estudio de casos. Técnicas que aparte de ayudar a acercar la realidad profesional y el desarrollo de competencias, contribuyen a revalorizar la formación práctica (López et al., 2016).

Otros resultados revelaron además que la mayoría de los egresados $(58,8 \%)$ consideraron no sentirse preparados para desempeñarse de manera apropiada en sus labores profesionales en las Big Four. Entre las causales que explican esta percepción se relatan principalmente los problemas para aplicar los conocimientos en la práctica de diversos contenidos del área tributaria y de auditoría. En específico, al ser consultados acerca de las temáticas concretas que le dificultaron su estadía en las auditoras mencionaron: la falta de dominio de ciertas normas propias, tales como: las Normas Internacionales de Información Financiera (NIIF), las Normas de Auditoría Generalmente Aceptadas (NAGA's), las Normas Internacionales de Auditoría (NIA) y las normas BEPS (Erosión de la Base Imponible y Transferencia de Utilidades); además del desconocimiento sobre la utilización del control interno desde la perspectiva del enfoque COSO (Committee of Sponsoring Organizations).

Dentro del mismo ámbito, casi la mitad de los encuestados $(47,1 \%)$ indicaron que para trabajar en las Big Four se necesita de un amplio dominio de las Normas Internacionales de Contabilidad (NIC), junto con los conocimientos suficientes sobre impuesto a la renta, franquicias, donaciones, convenios, etc. Adicionalmente, se les consultó si necesitaron realizar capacitaciones extras para obtener un nivel apropiado al cargo, donde el 35,3\% señaló que sí necesitó al menos de una capacitación en el área tributaria, mientras que otros necesitaron más de cinco capacitaciones o rendir al menos un diplomado.

Por último, entre las recomendaciones brindadas por los mismos egresados para suplir las deficiencias encontradas se cuentan: (i) potenciar el área de auditoría financiera; (ii) profundizar, como se mencionó anteriormente, conocimientos sobre aspectos tributarios y el uso de normas de la especialidad; y (iii) ahondar en la aplicación de aseveraciones de los estados financieros; y en la preparación de informes de auditoría. En la figura 4 se resumen los hallazgos antes señalados. 


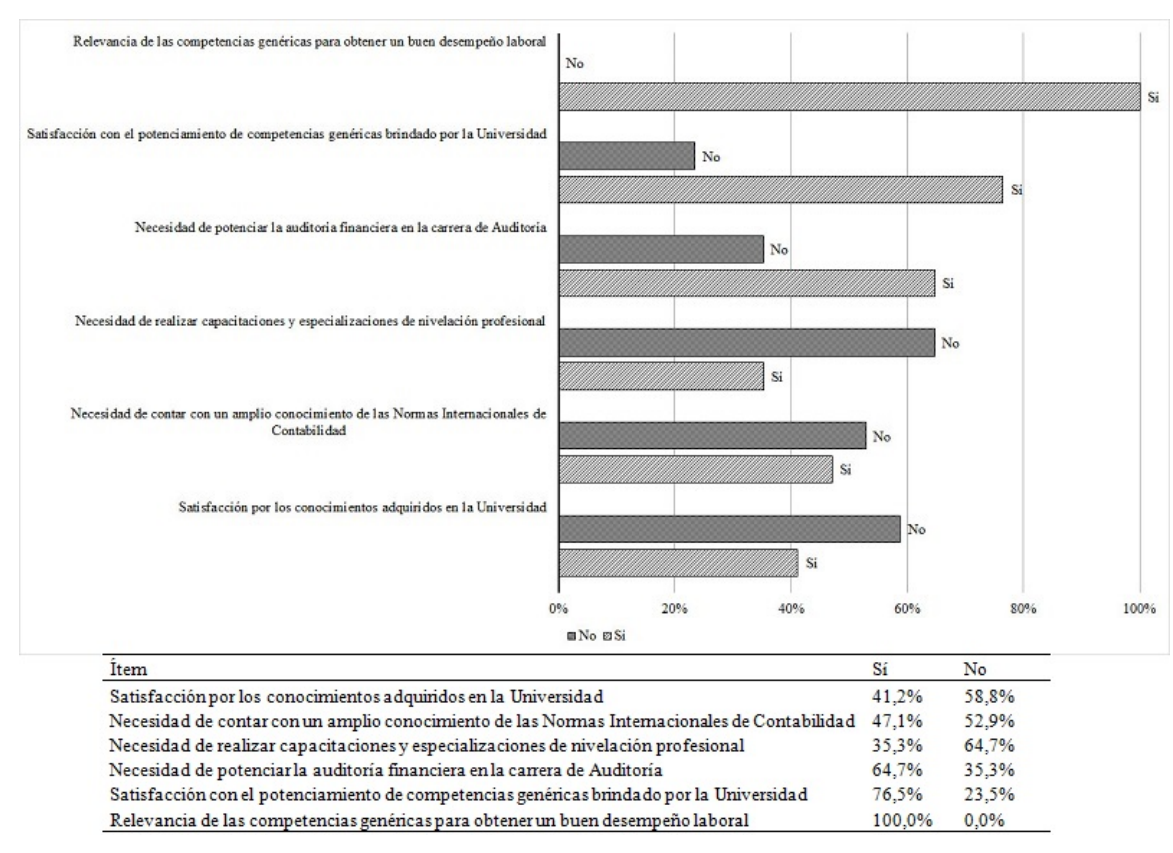

FIGURA 4

Percepción de los estudiantes egresados de la carrera de Auditoría sobre los conocimientos y habilidades adquiridas en su formación profesional y laboral. Fuente: elaboración propia.

\section{Competencias genéricas fundamentales para las Big Four y los estudiantes}

De los resultados obtenidos, destaca el alto valor que los empleadores de las cuatro grandes auditoras presentes en Chile otorgan a las competencias genéricas, particularmente, a las que Spencer \& Spencer (1993) denominan competencias directivas, competencias cognitivas y competencia de eficacia personal. Sin embargo, llama la atención que tanto las empresas como los colaboradores no manifestaran una orientación más explícita por competencias vinculadas a la responsabilidad social y compromiso ciudadano, o al compromiso con el medio ambiente, o con su medio-socio cultural.

En relación con lo expresado por los diferentes egresados de la carrera de Auditoría de la ULS, se devela que es fundamental el manejo de competencias genéricas tales como la capacidad para aplicar los conocimientos en la práctica, el trabajo en equipo, la autonomía, el saber enfrentar y solucionar problemas, demostrando capacidad de adaptación a situaciones complejas. Esto coincide con lo indicado en las entrevistas a los empleadores, los cuales además agregan como condición fundamental, que los colaboradores tengan ganas de aprender y constituir un real aporte para la organización, lo cual puede inclusive llevar a suplir otras deficiencias como la falta de preparación en ciertos contenidos teóricos.

Según lo antes mencionado, se confirma que los egresados y las auditoras convienen respecto a que es esencial contar con competencias genéricas, empero difieren en lo que concierne a la importancia relativa otorgada entre ellas. Los egresados se enfocan mayormente en las competencias derivadas de los conocimientos siendo estas percibidas como las fundamentales para tener éxito profesional, relegando el resto de las competencias como suplementarias para desarrollar un trabajo armonioso con sus pares. Los empleadores, en cambio, prestan principal atención en la búsqueda de personas con autonomía, capaces de solucionar problemas en el instante, además de poder trabajar en equipo, manifestando que los conocimientos son trascendentales, pero estos se pueden adquirir con el tiempo.

A modo de reflexión cabe resaltar que mientras las instituciones educativas no presten la atención suficiente en monitorear y entrenar habilidades genéricas claves como el trabajo en equipo y la comunicación, no podrá garantizarse el desarrollo de las habilidades y actitudes en los estudiantes que se consideran de mayor valor para desempeñarse exitosamente en el entorno cambiante de las organizaciones del siglo XXI (Adriaensen, Bijsmans \& Groen, 2019). 


\section{CONCLUSIONES}

El presente artículo tuvo por propósito explorar las competencias genéricas de los estudiantes de contabilidad y auditoría de Chile, requeridas por las cuatro firmas más importantes del sector de la consultoría y auditoría del mundo: las Big Four. Del análisis de los resultados se concluye que: (i) las condiciones del desempeño profesional del contador auditor en el presente exigen, además de las competencias específicas, el desarrollo de competencias genéricas que le permitan ejercer su rol eficientemente en contextos complejos, con autonomía, flexibilidad, integridad y compromiso; (ii) la formación de competencias genéricas son fundamentales para el buen desempeño profesional de los egresados de contabilidad y auditoría en las Big Four; (iii) entre las competencias genéricas fundamentales para las Big Four destacan las sistémicas (capacidad de aplicar los conocimientos en la práctica, capacidad de motivar, habilidad para trabajar de forma autónoma, capacidad de aprender y actualizarse continuamente), seguidas de competencias interpersonales (trabajo en equipo y habilidades interpersonales), y de competencias instrumentales (conocimientos sobre la profesión y capacidad de resolver problemas); (iv) A pesar de que hay coincidencia entre los empleadores y egresados respecto de las competencias genéricas fundamentales, existe de parte de los empleadores una mayor valoración (importancia relativa) por aquellas vinculadas a las competencias directivas y de eficacia personal (sistémicas e interpersonales); y (v) De las declaraciones de los egresados de Auditoría de la ULS se evidencian deficiencias en el desarrollo de competencias genéricas como la capacidad de aplicar los conocimientos en la práctica, habilidades interpersonales y de trabajo autónomo.

Los tópicos anteriores se pueden utilizar para que las autoridades de las instituciones educativas puedan rediseñar los programas de educación existentes y diseñar e implementar cursos que sean necesarios para facilitar el desarrollo de habilidades y conocimientos para una mejor inserción y desempeño del profesional contador auditor en organizaciones globales. Para ello es necesario un modelo que proporcione la integración y contextualización del desarrollo de habilidades genéricas, así como la provisión de un apoyo adecuado de los estudiantes de contador auditor durante todo su proceso de formación.

Por otra parte, para avanzar en este tema de estudio se sugiere la aplicación de la investigación desde un enfoque cuantitativo que mida la percepción sobre cada una de las competencias genéricas fundamentales para el contador auditor desde la integración de otras visiones (profesores, alumnos, titulados, reclutadores y empresas), indague el porqué de sus valoraciones y abarque un diseño metodológico bajo un tamaño muestral mayor complementado con otras técnicas investigativas de recolección de datos como el grupo focal y las entrevistas en profundidad. Asimismo, se hace interesante establecer un estudio comparativo con las competencias genéricas requeridas por otras auditoras, que no son las Big 4, para así verificar y detectar aquellas que son propias de su modelo de negocios y orientación estratégica.

Como reflexión final quedan varios cuestionamientos por atender, como por ejemplo: ¿cuál es la importancia que están dando las instituciones de educación superior al desarrollo de las competencias genéricas del estudiante contador auditor en Chile y Latinoamérica?, ¿qué competencias genéricas, bajo el contexto actual, se consideran fundamentales para el correcto ejercicio y desempeño profesional? ¿qué actividades de los programas curriculares están "planificadas" concretamente para desarrollarlas?, ¿de qué manera se está midiendo y controlando su desarrollo?, ¿cuentan los docentes del área contable con la metodología y didáctica adecuada para su enseñanza? La búsqueda de las respuestas a estas incógnitas permite comprender que lo anterior requiere de una planificación a mediano y largo plazo, que debe ser parte de una política institucional mayor que asigne claramente el tiempo y los recursos apropiados para la formación de un profesional integral, con capacidades, habilidades y valores alineados con los desafíos de la globalización.

\section{REFERENCIAS}

Abarca, N. (2016). Inteligencia Emocional en el Liderazgo. Santiago: El Mercurio. 
Adriaensen, J., Bijsmans, P., \& Groen, A. (2019). Monitoring Generic Skills Development in a Bachelor European Studies. Journal of Contemporary European Research, 15(1), 110-127. https://doi.org/10.30950/jcer.v15i1.1018

Amor, M., \& Serrano, R. (2019). The generic competences in the Initial Teacher Training. A comparative study among students, teachers and graduates of university education degree. Educación XXI, 22(1), 239-261. https://doi.o $\mathrm{rg} / 10.5944 /$ educxx1.21341

Avendaño-Castro, W., Rueda-Vera, G., \& Paz-Montes, L. (2016). La investigación formativa en las prácticas docentes de los profesores de un programa de contaduría pública. Cuadernos de Contabilidad, 17(43), 157-182. https:// doi.org/10.11144/Javeriana.cc17-43.ifpd

Bagley, P., Dalton, D., \& Ortegren, M. (2012). The factors that affect accountants' decisions to seek careers with big 4 versus non-big 4 accounting firms. Accounting Horizons, 26(2), 239-264. https://doi.org/10.2308/acch-50123

Bautista, I. (2016). Generic competences acquisition through classroom activities in first-year agricultural engineering students. International Journal of Educational Technology in Higher Education, 13(1). https://doi.org/10.1186 /s41239-016-0028-8

Beneitone, P., César, E., Gonzalez, J., Maleta, M., Siufi, R., \& Wagenaar, G. (2007). Reflexiones y perspectivas de la educación superior en América Latina. In Tuning Lationamérica. Recuperado de http://tuningacademy.org/w p-content/uploads/2014/02/TuningLAIII_Final-Report_SP.pdf

Boateng, A., \& Thompson, A. (2013). Study abroad Ghana: An international experiential learning. Journal of Social Work Education, 49(4), 701-715. https://doi.org/10.1080/10437797.2013.812897

Boude, O. (2011). Desarrollo de competencias genéricas y específicas en educación superior a través de una estrategia didáctica mediada por TIC en educación superior (II). Educación Médica Superior, 28(4), 652-666. Recuperado de https://dialnet.unirioja.es/servlet/tesis?codigo $=26307$

Bunney, D., Sharplin, E., \& Howitt, C. (2015). Generic skills for graduate accountants: the bigger picture, a social and economic imperative in the new knowledge economy. Higher Education Research and Development, 34(2), 256-269. https://doi.org/10.1080/07294360.2014.956700

Cabeza, L., Castrillón, J., \& Lombana, J. (2015). Competencias más importantes para la disciplina administrativa en Colombia. Contaduria y Administración, 60(4), 776-795.

Camargo-Escobar, I., \& Pardo-Adames, C. (2008). Competencias docentes de profesores de pregrado\#: diseño y validación de un instrumento de evaluación. Universitas Psychologica, 7(2), 441-456.

Carter, C., \& Spence, C. (2014). Being a Successful Professional: An Exploration of Who Makes Partner in the Big 4. Contemporary Accounting Research, 31(4), 949-981. https://doi.org/10.1111/1911-3846.12059

Contreras, J. (2011). Formación de competencias: tendencias y desafíos en el siglo XXI. Universitas, Revista de Ciencias Sociales y Humanas, (15), 109-138. Recuperado a partir de http://www.redalyc.org/html/4761/47614738300 5/

Corominas-Rovira, E. (2001). Competencias Genéricas en la formación universitaria. Revista de Educación, 325(2001), 299-321.

Crawford, L., Helliar, C., \& Monk, E. A. (2011). Generic skills in audit education. Accounting Education, 20(2), 115-131. https://doi.org/10.1080/09639284.2011.557487

de Pablos, J. (2010). Universidad y sociedad del conocimiento. Las competencias informacionales y digitales. Revista de Universidad y Sociedad Del Conocimiento, 7(2), 6-16. Recuperado a partir de http://rusc.uoc.edu/ojs/index. $\mathrm{php} /$ rusc/article/view/v7n2-de-pablos/v7n2-de-pablos

Dextre, J. (2013). Los retos de la formación por competencias del contador público/The challenges of competency based education of Certified Public Accountant. Contabilidad y Negocios, 8(16), 35-47. Recuperado a partir de http://search.proquest.com/docview/1507220501 ?accountid=34489\%5Cnhttp://atoz.ebsco.com/titles.as p?lang $=\&$ lang.subject $=\&$ lang $\cdot \mathrm{menu}=\& \mathrm{KW}=\& \mathrm{sid}=258657917 \& \mathrm{id}=1811 \& \mathrm{SF}=\mathrm{Titles} \& S \mathrm{ST}=$ Contains $\& \mathrm{cmd}$ SearchSubmit $=$ BuscarContabilidad $+\mathrm{y}+$ Negocios/

Durán, M., Mejía, M., \& Reyes, A. (2012). Competencias genéricas y profesionales\#: un enfoque analítico de la licenciatura en contaduría. XVII Congreso Internacional de Contaduría, Administración e Informática.UNAM. Recuperado de http://premio.investiga.fca.unam.mx/docs/ponencias/2012/8.1.pdf 
Esquetini, C., Becerra, M., Cisternas, B., et al. (2013). Educación Superior en América Latina: reflexiones y perspectivas en Administración. Bilbao: Universidad de Deusto.

Fernandez-Feijoo, B., Romero, S., \& Ruiz, S. (2018). Financial Auditor and Sustainability Reporting: Does it matter? Corporate Social Responsibility and Environmental Management, 25(3), 209-224. https://doi.org/10.1002/csr. 1449

Fernández, L. (2010). Soft skills, cualidades personales, "competencias no técnicas", etc. ReVisión, 3(1), 1-3.

Gil, J. (2007). La evaluación de competencias laborales. Educación XX1, (10), 83-106. https://doi.org/10.5944/edu $\operatorname{cxx} 1.1 .10 .298$

González, V., \& González, R. (2008). Competencias Genéricas y Formación Profesional\#: Un Análisis desde la Docencia Universitaria. Revista Iberoamericana de Educación, 47, 185-209. https://doi.org/10.1016/j.estger.2 014.12 .005

Kallioinen, O. (2010). Defining and Comparing Generic. European Educational Research Journal, 9(1), 56-68. https ://doi.org/10.2304/eerj.2010.9.1.56

López, C., Benedito, V., \& León, M. J. (2016). El Enfoque de Competencias en la Formación Universitaria y su Impacto en la Evaluación: La Perspectiva de un Grupo de Profesionales Expertos en Pedagogía. Formación Universitaria, 9(4), 11-22. https://doi.org/10.4067/S0718-50062016000400003

Lyudmila, K., Alexey, S., \& Sevinj, A. (2019). Audit as a key tool for business knowledge management system research. In D. D. Muslim Ibrahimov, Ana Aleksic (Ed.), 37th International Scientific Conference on Economic and Social Development - "Socio Economic Problems of Sustainable Development" (pp. 14-15). Baku.

Miró, J., \& Jaume-i-Capó, A. (2010). Repositorio de actividades para enseñar competencias transversales repository of activities to teach transferable competences. Revista de Docencia Universitaria, 8(1), 101-110. https://doi.org/ https://doi.org/10.4995/redu.2010.6219

Miyakawa, E., Kawakubo, A., \& Oguchi, T. (2019). Do people who travel more perform better at work? International Journal of Tourism Research, jtr. 2269. https://doi.org/10.1002/jtr.2269

Montilla, O. de J., \& Herrera, L. (2006). El deber ser de la Auditoría. Estudios Gerenciales, 22(98), 83-110. Recuperado de http://search.ebscohost.com/login.aspx?direct=true\&db=fua\&AN=22465961\&lang=es\&site=ehost-live

Navarro, G., Rubio, V., Lavado, S., Minnicelli, A., \& Acuña, J. (2017). Razones y propósitos para incorporar la responsabilidad social en la formación de personas y en organizaciones de Latinoamérica. Revista Latinoamericana de Educación Inclusiva, 11(2), 51-72. https://doi.org/10.4067/S0718-73782017000200005

OCDE - Organisation for Economic Co-Operation and Development. (2001). Knowledge and Skills for Life First results from the OECD Programme for International Student Assessment (PISA) 2000. Recuperado a partir de ht tp://www.oecd.org/education/school/programmeforinternationalstudentassessmentpisa/33691596.pdf

Ospina, Y. (2014). Rescatar lo antropológico... Una necesidad de la educación. Praxis \& Saber, 5(10), 193-218. Recuperado a partir de http://www.scielo.org.co/pdf/prasa/v5n10/v5n10a11.pdf

Parlamis, J., \& Monnot, M. (2018). Getting to the CORE: Putting an End to the Term "Soft Skills." Journal of Management Inquiry, 1-3. https://doi.org/10.1177/1056492618818023

Peralta, L., \& Piña, M. (2008). El contador auditor\#: desafíos de una formación personal y profesional en un mundo globalizado. CAPIC REVIEW, 6, 21-34. Recuperado a partir de http://www.capic.cl/wp-content/uploads/20 15/10/vol6art2.pdf

Pérez, J. , Espinoza, C., \& Peralta, B. (2016). La Responsabilidad Social Empresarial y su Enfoque Ambiental: Una visión sostenible a futuro. Revista Universidad y Futuro, 8(3), 169-178. Recuperado de http://scielo.sld.cu/pdf /rus/v8n3/rus23316.pdf

Rodríguez, H. (2007). El paradigma de las competencias hacia la educación superior. Revista de La Facultad de Ciencias Económicas de La Universidad Militar Nueva Granada, 15(1), 145-165.

Roncancio, Á., Mira, G., \& Muñoz, N. (2017). Las competencias en la formación del profesional contable: una revisión de las posturas institucionales y educativas en Colombia. Revista Facultad de Ciencias Económicas, 25(2), 83-103. https://doi.org/10.18359/rfce.3070 
Rubau, C., Tovar, M., \& Fernández, A. (2013). Importancia de las competencias genéricas Tuning-América Latina para el desarrollo de la formación para la investigación. Estudio comparativo en el Instituto de Ciencias Básicas de la Universidad Nacional de Cuyo (Argentina). IX Congreso Internacional Sobre Investigación En Didáctica de Las Ciencias, 3114-3119. Recuperado a partir de https://www.raco.cat/index.php/Ensenanza/article/view/30828 $1 / 398291$

Rullan, M., Fernández, M., Estapé, G., \& Márquez, D. (2010). La evaluación de competencias transversales en la materia trabajos fin de grado. REDU. Revista de Docencia Universitaria, 8(1), 74-100. https://doi.org/10.4995/redu.2 010.6218

Sánchez, G. (2006). Auditoría de estados financieros (Segunda). México: Pearson Education.

Shah, M., \& Prakash, A. (2018). Developing generic competencies for infrastructure managers in India. International Journal of Managing Projects in Business, 1-17. https://doi.org/10.1108/IJMPB-03-2017-0030

Snellman, C. (2015). University in Knowledge Society\#: Role and Challenges. Journal of System and Management Sciences, 5(4), 84-113. Recuperado de https://www.researchgate.net/publication/308368679_University_in_ Knowledge_Society_Role_and_Challenges

Spencer, L., \& Spencer, S. (1993). Compentence at work. Models for superior Performance. Nueva York: Wiley \& Sons.

Tejeda, R. (2017). Las competencias transversales, su pertinencia en la integralidad de la formación de profesionales.Didasc@lia: Didáctica y Educación, 7(2016), 199-228. Recuperado de https://www.researchgate.net/publication/320133699_las_competencias_transversales_su_pertinencia_en _la_integralidad_de_la_formacion_de_profesionales

Tobón, S. (2005). Formación basada en competencias. Pensamiento complejo, diseño curricular y didáctica. Ecoe Ediciones, 310. Recuperado de https://www.uv.mx/psicologia/files/2015/07/Tobon-S.-Formacion-basada-encompetencias.pdf\%0Ahttp://bcnslp.edu.mx/antologias-rieb-2012/preescolar-i-semestre/DFySPreesco/Materi ales/UnidadA1_DFySpreesco/RecursosExtra/Tob\%F3nFormaci\%F3nBasadaC05.pdf

Ureña, E., \& Ureña, V. (2016). Las competencias genéricas en la formación del estudiantado de la enseñanza del inglés en la Universidad de Costa Rica: Visión de docentes y estudiantes y su relación con las demandas del sector empleador. InterSedes, XVII(36), 2-33. Recuperado de http://www.redalyc.org/articulo.oa?id=66648525005

Villarroel, V., \& Bruna, D. (2014). Reflexiones en torno a las competencias genéricas en educación superior: Un desafío pendiente. Psicoperspectivas, 13(1), 23-34. https://doi.org/10.5027/PSICOPERSPECTIVAS-VOL13SSUE1FULLTEXT-335

\section{Notas}

* Artículo de investigación científica.

\section{Licencia Creative Commons CC BY 4.0}

Para citar este articulo: Araya-Pizarro, S. C. (2019). Competencias genéricas de los estudiantes de Auditoría requeridas por las Big Four en Chile. Cuadernos de Contabilidad, 20(49). DOI:https://doi.org/10.11144/ Javeriana.cc20-49.cgea 